\title{
Detection and characterization of water ingress in honeycomb structures by passive and active infrared thermography using a high resolution camera
}

\author{
by C. Ibarra-Castanedo*, F. Marcotte ${ }^{* *}$, M. Genest ${ }^{+}$, L. Brault ${ }^{*}$, V. Farley ${ }^{*}$ and X. P. V. Maldague \\ ${ }^{\star}$ Computer Vision and Systems Laboratory, Department of Electrical and Computer Engineering, 1065, av. de la \\ Médecine, Laval University, Quebec City, Canada, G1V 0A6; \{Bendada, IbarraC, MaldagX\}@gel.ulaval.ca \\ ** Telops, 100-2600 avenue St-Jean-Baptiste, Quebec (Quebec) Canada G2E 6J5 \\ ${ }^{+}$National Research Council Canada, 1200 Montreal Road, Bldg. M-14, Room 130, Ottawa (ON) Canada K1A OR6.
}

\begin{abstract}
Honeycomb sandwich structures are an attractive choice for the production of lightweight and resistant aeronautical components. These multilayered materials are however susceptible to water ingress leading sometimes to structural damage. Passive thermographic inspection is an interesting technique that can be applied right after landing, when water inside the honeycomb structure is often frozen producing large temperature gradients. Alternatively, an active configuration can be considered when inspection is performed several minutes after landing. In this paper, the passive approach was investigated on an aeronautical part where water ingress was simulated. A high-definition camera and a microbolometer were used for comparison.
\end{abstract}

\section{Introduction}

Sandwiched structures combining aluminum honeycomb cores between carbon fibre composite skins has been a popular choice for military and civil aerospace components for several years. The interest for this kind of structure comes from its characteristics combining lightweight, low cost, fatigue resistance and high strength and stiffness [1]. It is well-known however, that these structures are often affected by water ingress (among other problems) with a potential risk of aluminum core corrosion and/or adhesive bond (node and skin-to-core) degradation [2]-[4]. Pressure and temperature variations during take-off and landing produce considerable stress on honeycomb aircraft structures, promoting moisture or water ingress by different ways [5]: capillarity along cracks and adhesives; wicking along fiber matrix interfaces; transport from the environment into the honeycomb core through damaged areas, e.g. around fasteners, voids, cracks or unsealed joints; or diffusion through fibers and adhesives. Previous injection and potted repairs have also been cited as moisture diffusion promoters [6]. The presence of water or other fluids such as kerosene, hydraulic fluid and de-icing agents in any of its forms (gas vapor, liquid or ice) promotes corrosion, cell breakage, node bond failure and induce composite layer delaminations and skin disbonds [7]. Furthermore, standing water (in liquid or vapor state) trapped in the structure could cause structural damage (node bond failure) due to repeated freeze-thaw cycles due to normal flight operations [1], [8]. In a study involving 15 Boeing 767 aircraft, it was found that these airplanes could contain up to $40 \mathrm{~kg}$ of water mostly in the external honeycomb composite panels [9].

Several nondestructive techniques for water ingress control have been proposed. The hot-water leak test is a classic technique in which honeycomb sandwich structures (after fabrication or repair) are inspected for leakage paths, by immersing them in tanks with hot water. The appearance of air bubbles provides an indication of the presence of leaks [5]. This approach has the disadvantage of potentially encourage water ingress into the components. This problem has been recently addressed using a helium mass spectrometer leak detector, in which the specimen is placed inside a bag filled with helium and a spectrometer is employed to detect escape points [10]. NDT techniques that actually detect water are also available, including ultrasonic testing [11]; x-ray and neutron radiography [8] and of course infrared thermography [12]-[14].

Active infrared thermography is fast and relatively easy to deploy technique allowing detection of areas affected by water in aerospace sandwiched components showing excellent agreement with ultrasounds and radiography inspections [11]. On the other hand, passive assessment of aircrafts has also been explored. This is performed right after landing to take advantage of a large temperature gradient between the water inside the structure, usually frozen, and the ambient temperature [9], [15]. In this research, the advantages of using a high resolution infrared camera in passive thermography are investigated. 


\section{Experimental setup}

The investigated specimen consists of a section of a military aircraft component made of an aluminum honeycomb core sandwiched between two carbon fibre skins. Eight (8) holes were produced on the back side of the sample in order to have easy access to the core cells as shown in Fig. 1a. The cells inside these areas were filled with different amounts of water using a graduated syringe (see Fig. 1a). Either one or ten cells were filled with water as indicated in Table 1, which provides the exact water volumes used in each case.

The specimen was then introduced in a cold chamber (see Fig. $1 \mathrm{~b}$ ) until a temperature of $-20^{\circ} \mathrm{C}$ was reached and held for 10 minutes before taking it out to start the experiment. Once the specimen was in position, the surface temperature was monitored using four different IR cameras as seen in Fig. 1c: a high definition mid-wave IR camera (Telops HD-IR 1280, $1280 \times 1024$ pixels, InSb, 3.6-4.9 $\mu \mathrm{m}$ ), a mid-wave IR camera (TEL-1000 MW, 640×512 pixels, InSb, 3.6-4.9 $\mu \mathrm{m}$ ), a long-wave IR camera (TEL-1000 LW, 640×512 pixels, HgCdTe, 8-9.6 $\mu \mathrm{m}$ ) and a microbolometer (Jenoptik IR-TCM 384, 384x288 pixels, 7.5-14 $\mu \mathrm{m})$. In this paper however, results exclusively from the HD camera and the microbolometer are presented and discussed.

A second set of experiments were carried out in order to evaluate the performance of a high definition camera for the detection of water, in liquid and solid states, inside the honeycomb structure. For this, four (4) honeycomb zones (A, D, E and $\mathrm{H}$ ) were filled with different amounts of water as described in Table 2, and left on a cold chamber following a similar procedure as described previously for several hours until water was completely frozen. The specimen was then removed from the cold chamber and the data acquisition started. A total of 1920 thermograms were obtained for both cameras at a frame rate of $1 \mathrm{~Hz}$, hence the observation time lasted 32 minutes.

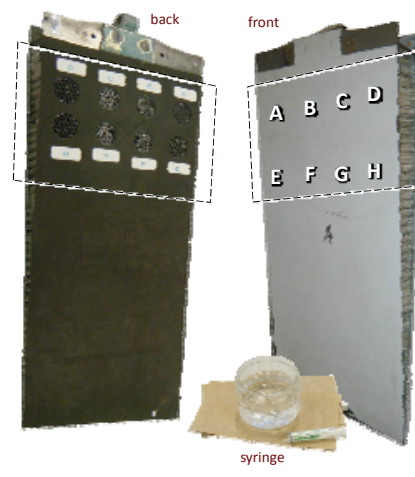

(a)

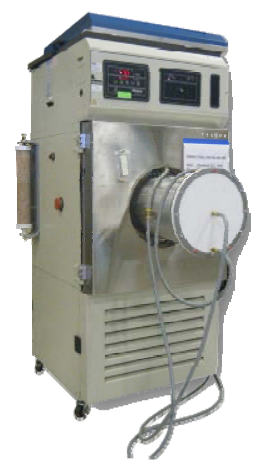

(b)

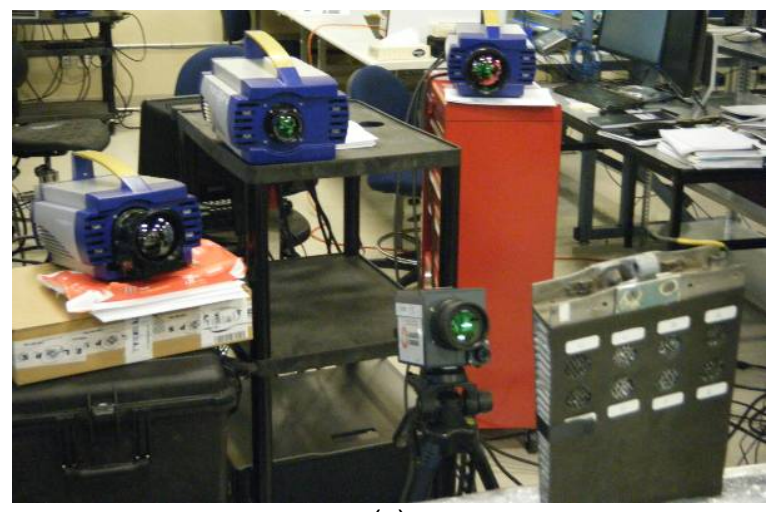

(c)

Fig. 1. (a) Front and back sides of the specimen (military aircraft component) and graduated syringe used to fill up cells with different amounts of water; (b) cold chamber allowing to cool down the specimen down to $-20^{\circ} \mathrm{C}$; and (c) view of the experimental setup with four cameras from left to right: MW, LW, microbolometer and HD.

Table 1. Description of defect areas.

\begin{tabular}{c|cccccccc} 
Defect & A & B & C & D & E & F & G & H \\
\hline Number of cells filled with water & 10 & 1 & 1 & 10 & 10 & 1 & 1 & 10 \\
Water volume [mL] & 9 & 0.4 & 0.8 & 4 & 2 & 0.2 & 0.6 & 6 \\
Maximum contrast [arbitrary] & 699 & 281 & 413 & 735 & 540 & 147 & 302 & 761 \\
Time for maximum contrast [s] & $>1200$ & 455 & 598 & 1091 & 750 & 336 & 502 & 1180 \\
Ending observation time [s] & $>1200$ & 665 & 847 & $>1200$ & 986 & 487 & 693 & $>1200$ \\
\cline { 2 - 8 }
\end{tabular}

Table 2. Defect description for the second set of experiments.

\begin{tabular}{c|cccc|} 
Defect & A & D & E & $H$ \\
\hline $\begin{array}{c}\text { Number of cells filled with water } \\
\text { Water volume }[\mathrm{mL}]\end{array}$ & 1 & 5 & 10 & 1 \\
0.9 & $1(5 \times 0.2)$ & $9(10 \times 0.9)$ & 0.2 \\
\cline { 2 - 5 }
\end{tabular}




\section{Experimental results and discussion}

\subsection{The impact of water volume}

Fig. 2 shows three thermograms obtained with the HD camera at different times. Fig. 2a was obtained $295 \mathrm{~s}$ after the acquisition started, i.e. the specimen had almost 5 minutes of warming up at this point and all defects can be seen. However, defect $F$ is no longer seen $518 \mathrm{~s}$ (8.6 min) after starting the acquisition (see Fig. 2b). As seen in Table 1, defect $F$ has the lowest water volume of all and a single cell was used. All other defects loss thermal contrast at different times depending on the water volume. After 1315 s, i.e. almost 22 minutes after starting the experiment, only the three larger defects, i.e. A, H and D (9, 6 and $4 \mathrm{ml}$, respectively), can still be observed (see Fig. $2 \mathrm{~b}$ ).

The temperature profiles calculated from the average value of a $5 \times 5$ pixel window inside defective areas are presented in Fig. 3a. Similarly, the temperature profile for a sound area (non-defective, see Fig. 2c) is shown as well. As can be seen, temperature evolution for a non-defective area behaves differently than defective ones. The sound area warms up following a logarithmic growth with respect to time. In the presence of water though, temperature profiles diverge from this behavior (since it takes longer to warm up water). It is apparent from this graphic that the divergent time for all defects is approximately the same (roughly around $180 \mathrm{~s}$ ), regardless of the water extend and volume. Nevertheless, defects temperature profiles gradually merge with the sound area temperature profiles at times that are directly dependent on the volume of water contained in the cells: larger defects profiles merge later than smaller defects. This merging time is in fact the time at which defect a particular loses its thermal contrast with respect to a non-defective area or in other words, the defect disappears at this point from the thermogram sequence. The merging time can be determined from these curves for defects under $2 \mathrm{ml}$ of water. Temperature profiles for defects having volumes greater than $2 \mathrm{ml}$ took longer than 20 minutes to loose thermal contrast (for which no data is available).

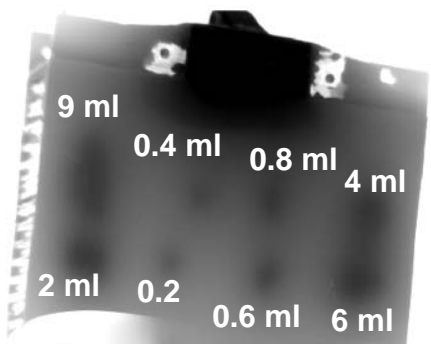

(a)

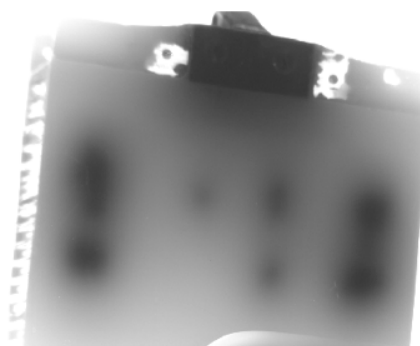

(b)

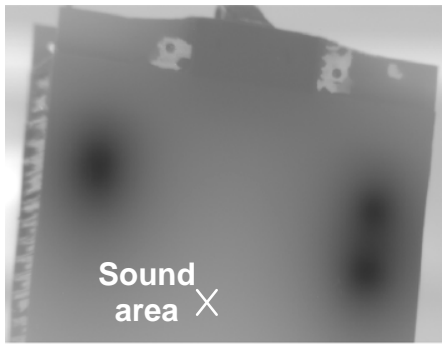

(c)

Fig. 2. Defect signature evolution through time: (a) Early thermogram at $t=295 \mathrm{~s}$ showing all defects (A to $H$ ); (b) thermogram at $t=518 \mathrm{~s}$ showing all defects except defect $\mathrm{F}$; (c) thermogram at $t=1315$ s showing only defects $\mathrm{A}, \mathrm{D}$ and $\mathrm{H}$.

Temperature contrast profiles can be reconstructed from the temperature profiles in Fig. 3a by subtracting the defect temperature value from the sound area temperature.

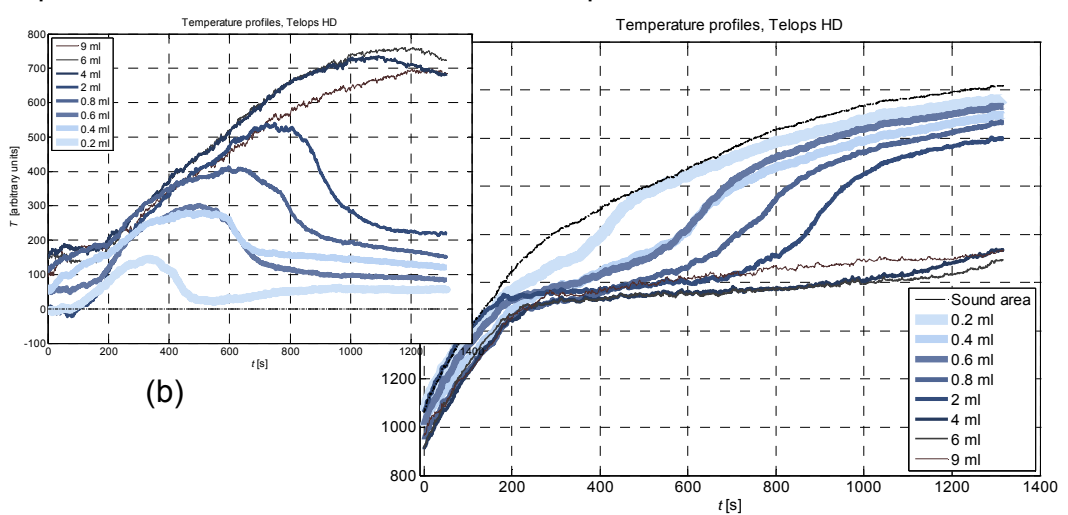

(a)

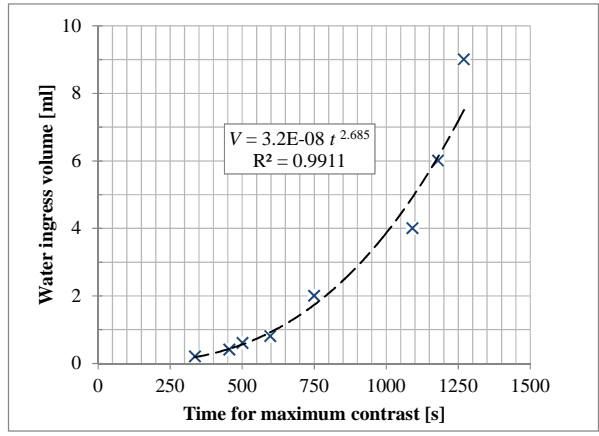

(c)

Fig. 3. (a) Temperature profiles over a sound area (dotted line) and the defect areas (solid lines), calculated using the average value of a $5 \times 5$ pixels window inside the areas of interest; (b) temperature contrast profiles calculated as the difference between the sound area and the defect profiles shown in (a); and Correlation between time for maximum temperature contrast and defect volume. 
The resulting profiles are presented in Fig. 3b. It should be noted that there is an offset between the initial thermal contrasts for the different profiles. This is due to the fact that the sound area was chosen arbitrarily, in between defects $F(0.2$ $\mathrm{ml})$ and $\mathrm{G}(0.6 \mathrm{ml})$ in this case. Hence, the temperature contrast of defects $F$ and $\mathrm{G}$ starts and ends close to zero, but there is an offset for all other defects. This situation would be different if a different sound area was chosen of course. However, this variability has little impact on the determination of the end of contrast times, when defective temperature profiles merge to non-defective ones. These times are presented in the last column of Table 1.

Additionally, the contrast profiles present a maximum value at a time that depends on the water volume. The maximum temperatures and the corresponding times are also presented in Table 1.

From Fig. $3 \mathrm{a}$ and $\mathrm{b}$, it can be observed that there is a direct relationship between the time of maximum contrast $t_{\text {max }}$, and the corresponding volume of water $V$, which can be confirmed by gathering all data as shown in Fig. 4 . As can be seen, experimental data of $V$ vs. $t_{\max }$, fit properly to a power function. This equation was obtained by fitting all the experimental data with the exception of the $9 \mathrm{ml}$ defect for which the maximum temperature was not reached at the end of the acquisition (> 20 minutes). In fact, according to the obtained correlation, the time for maximum contrast for a volume of $9 \mathrm{ml}$ should be around $1440 \mathrm{~s}$ (24 $\mathrm{min})$ after warming up starts.

Some important conclusions can be derived from these results. Firstly, the early time for defect contrast is probably not the best parameter to quantify water ingress in honeycomb structures since it is practically the same for all the defects studied herein, i.e. for water volumes from 0.2 to $9 \mathrm{ml}$. Secondly, the time of maximum temperature contrast seems more appropriate since it is practically unaffected by contrast variations dues to sound area selection. Furthermore, there is an excellent correlation between the time of maximum contrast and water volume that can be represented with a power function for the water quantities studied herein. Lastly, there exists a limited time to passively detect water ingress in honeycomb structures after landing, which is particularly important for small defects. For instance, a water volume of $0.2 \mathrm{ml}$ will have a maximum temperature contrast roughly 6 minutes after landing (assuming that warming up starts while in the ground). The time for maximum contrast increase to 10 minutes for $0.8 \mathrm{ml}$ of water and to 20 minutes for $6 \mathrm{ml}$ of water, as seen in Table 1 . Furthermore, the ending defect contrast time is also relatively limited for small defects, e.g. for a $0.2 \mathrm{ml}$ defect, contrast ends 8 minutes after landing, whilst for a $2 \mathrm{ml}$ it lasts for 16 minutes. Hence, depending on the time required for a plane to land and taxiing to its final position, it will be possible to detect and/or characterize water ingress of more or less severity.

\subsection{The impact of the distance from the target}

\subsubsection{Camera to target distance of $1.5 \mathrm{~m}$}

The specimen was inspected simultaneously employing a high definition (HD) camera (MWIR 1280x1024 pixels, $100 \mathrm{~mm}$ lens) and a microbolometer (LWIR 680 x 480 pixels, $20 \mathrm{~mm}$ lens). Two experiments were carried out using two different distances (camera to target), the firs one at $1.8 \mathrm{~m}$, and the second one at $18 \mathrm{~m}$. Fig. 4 shows the field-of-view (FOV) obtained from both cameras for the first test $(1.8 \mathrm{~m})$. The temperature bar (in Kelvins) is shown for the HD camera (Fig. 4a) for reference. The regions-of-interest (ROI) are also indicated.

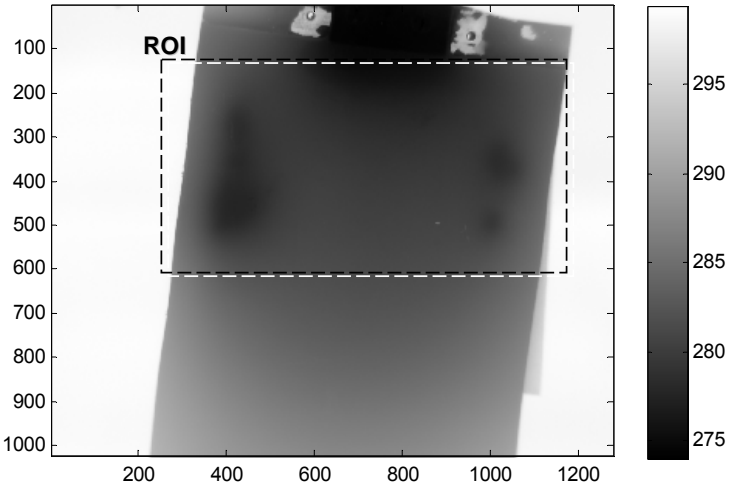

(a)

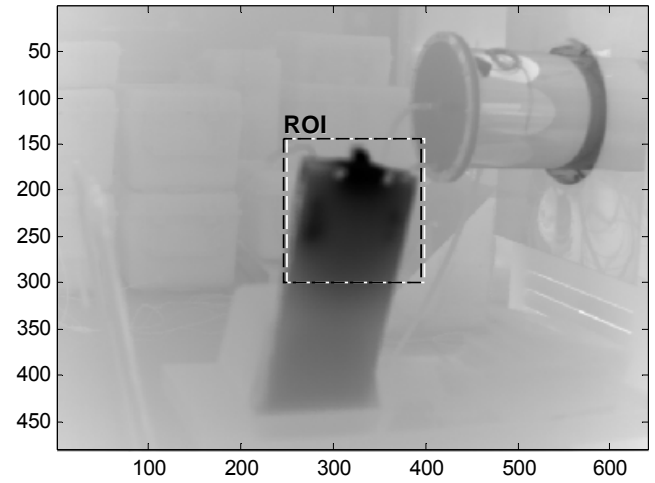

(b)

Fig. 4. FOV during data acquisition for target at $1.8 \mathrm{~m}$ : (a) high definition camera with $100 \mathrm{~mm}$ photo lens; and (b) microbolometer with $20 \mathrm{~mm}$ lens.

The four defects can be seen in these images, although they are better resolve in the case of the HD camera as expected. The temperature profiles for the four defective zones are presented in Fig. 5, they all warm up following a quasilogarithmic growth. Nevertheless, defective areas (with frozen water) diverge from non-defective curve (the warmest profile) for an interval that is related to the volume of water present in the cells. It is observed that defects containing less water warm 
up at a faster rate than those having a greater amount of water. However, this observation is not completely true in the case of Defect $A$, which contains $0.9 \mathrm{ml}$ of water. With this volume of water it is supposed to be slightly warmer than Defect $D$ (filled with $1 \mathrm{ml}$ of water). A possible explanation for this behaviour could be devised by the fact that, in Defect A, only 1 cell was filled with water $(0.9 \mathrm{ml})$, whilst 5 cells were filled with $0.2 \mathrm{ml}$ each for Defect $D$. Hence, even though the amount of water is approximately the same for both defect areas, the thermal signature is stronger for a single cell containing a greater amount of water $(0.9 \mathrm{ml}$ vs. $5 \times 0.2 \mathrm{ml})$. Another possibility is that water has migrated to Defect A (possibly from Defect $\mathrm{H}$, which is the closest and was filled with a large amount of water) during the preparation of the defects and/ or during data acquisition as the water melted. Hence, the actual volume of water in Defect A could be actually greater than $0.9 \mathrm{ml}$. This second alternative is plausible considering that the thermal signature for Defect $\mathrm{H}$, with a single cell filled with $0.2 \mathrm{ml}$, is considerably warmer than for Defect A.

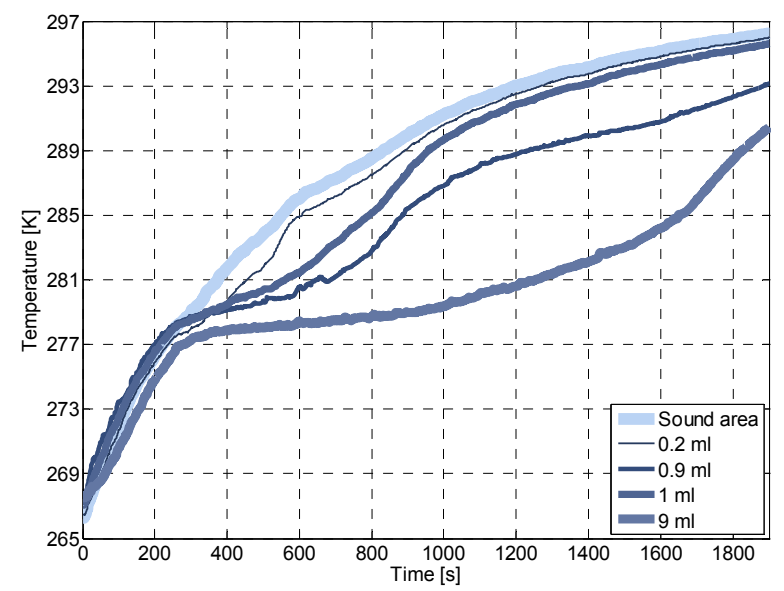

Fig. 5. Temperature profiles for the 4 defective areas, from high definition camera.

Fig. 6 present a comparison between images from the HD camera (left column) and the microbolometer (right column). Raw data (top row) show all four defects with best contrast for the HD camera. Advanced thermographic signal processing allows to increase contrast even further for both cameras. Principal component thermography [16] results, although similar for both cameras, they present striking differences in spatial resolution between them.

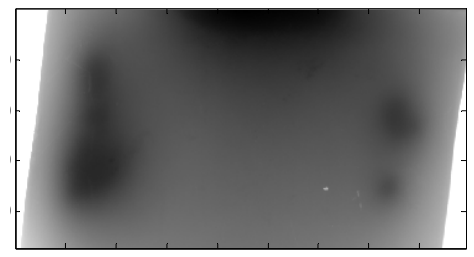

(a)

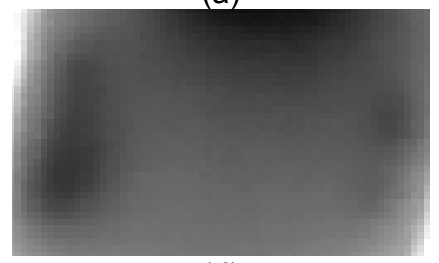

(d)

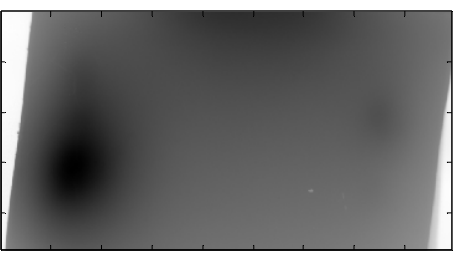

(b)

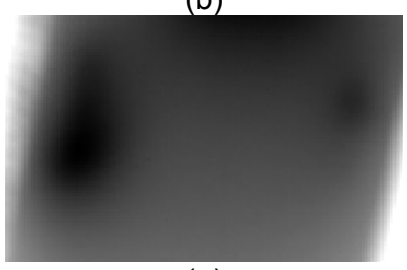

(e)

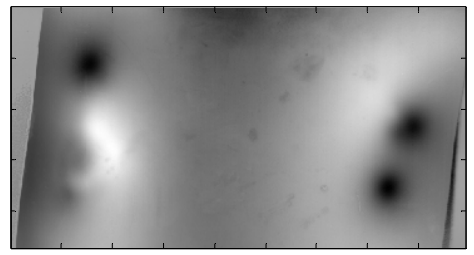

(c)

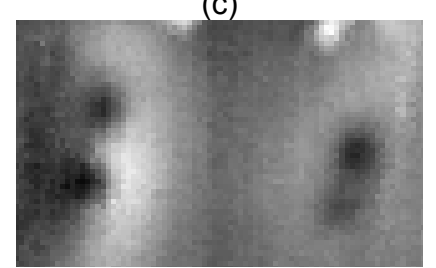

(f)

Fig. 6. Data processing results target at $1.8 \mathrm{~m}$. Top row (high definition camera): (a) raw thermogram at $t=6.5 \mathrm{~s}$; and PCT results (b) EOF1 and (c) EOF5. Bottom row (microbolometer right): (d) raw thermogram at $t=6.5 \mathrm{~s}$; and PCT results (b) EOF1 and (c) EOF8.

\subsubsection{Camera to target distance of $18 \mathrm{~m}$}

As mentioned before, a second test using the same defect configuration was repeated, this time however from a distance of $18 \mathrm{~m}$ (camera to target). Fig. 7 show two selected thermograms with the complete FOV of both cameras. In the case of the HD camera, a circular pattern is noticed at the center or the image. This is due to the relatively long integration 
time $(8.8 \mathrm{~ms})$ used for this experiment. This value was chosen in order to increase sensitivity at low temperatures (belowclose to zero centigrade).

Results in Fig. 8 portray the impact of spatial resolution and sensibility for defect definition at this distance (18 $\mathrm{m}$ ). The top row presents the HD images, the first result in this row corresponds to the raw thermogram (no processing) at a particular time $(t=469 \mathrm{~s})$, in which the four defective areas can be clearly seen. PCT processing helps to increase defect contrast even further as can be seen in the results of the second and third column. Microbolometer results are presented at the bottom line of this figure as reference. The raw thermogram corresponds to the same time as the corresponding HD image, and PCT results were chosen (by visual examination) as they presented the best defect contrast. Only two defective areas can be devised from these images corresponding to defects $E$ and $D$, that is 9 and $1 \mathrm{ml}$ of water, respectively.

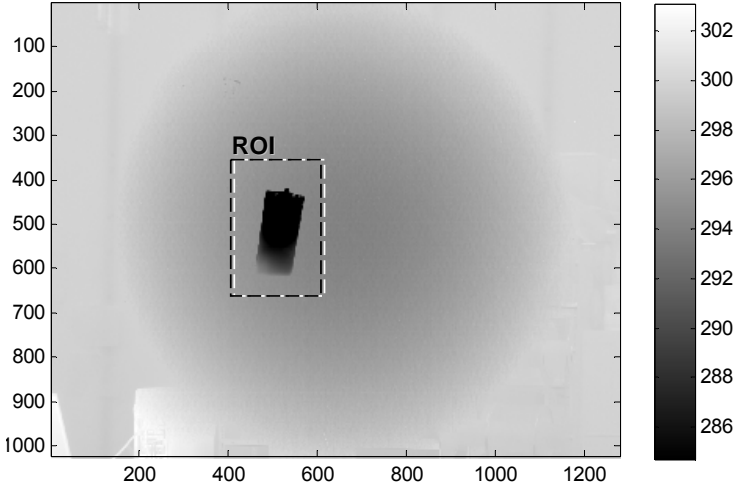

(a)

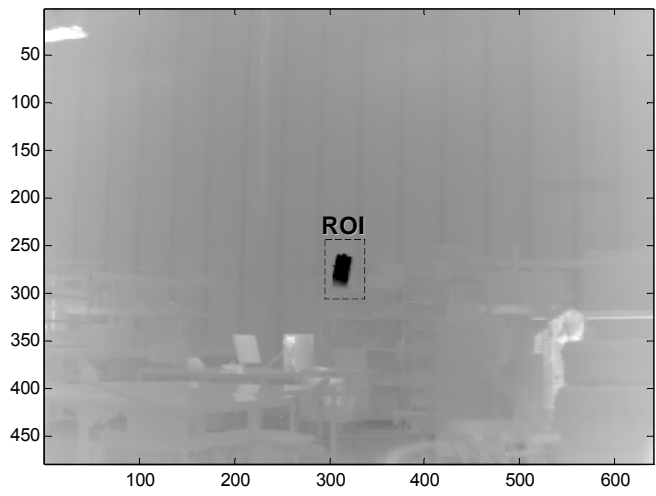

(b)

Fig. 7. FOV during data acquisition for target at $18 \mathrm{~m}$ : (a) high definition camera with $100 \mathrm{~mm}$ photo lens; and (b) microbolometer with $20 \mathrm{~mm}$ lens.

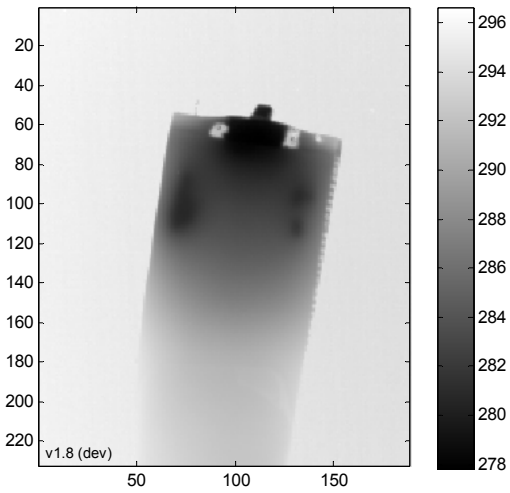

(a)

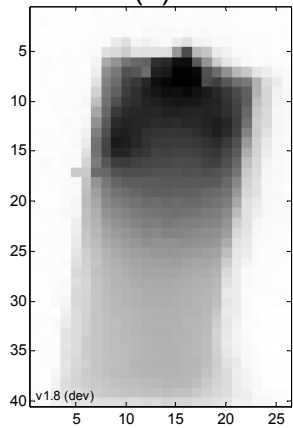

(d)

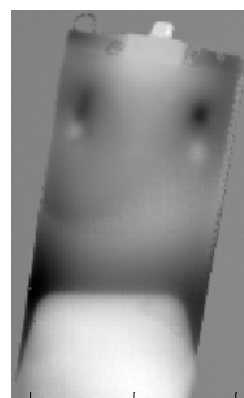

(b)

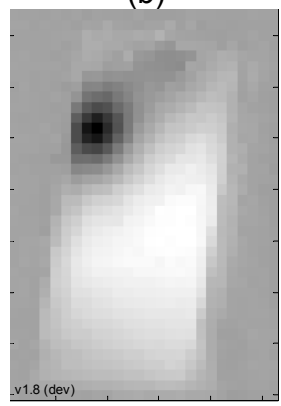

(e)

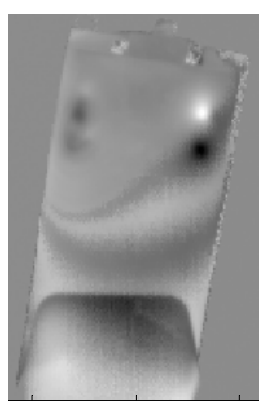

(c)

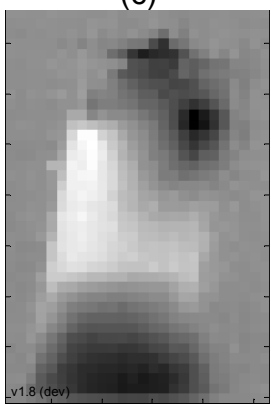

(f)

Fig. 8. Passive inspection with a camera-target distance of $18 \mathrm{~m}$. Top row (HD camera): (a) Raw thermogram at $t=469 \mathrm{~s}$; and PCT results (b) EOF4 and (c) EOF8. Bottom row (microbolometer): (d) Raw thermogram at $t=469 \mathrm{~s}$; and PCT results (e) EOF1 and (f) EOF3. 
Hence, it can be said that, while all defects (containing from 0.2 to $9 \mathrm{ml}$ of water) were clearly detected using a HD camera (even without processing), spatial resolution was simply not enough to distinguish all four defects using a microbolometer at $18 \mathrm{~m}$ from the sample. The thermal signatures of two areas, apparently corresponding to defects $E$ and $D$, are devised in the microbolometer processed results (Fig. 8e and f).

\subsection{Active inspection after $\mathbf{3 0}$ minutes}

\subsubsection{Camera to target distance of $1.5 \mathrm{~m}$}

Active thermography was also investigated at the end of the passive experiment (after 32 minutes). A heat gun was also employed as a heating source for defect detection at the end of the cycle of the $1.8 \mathrm{~m}$ camera-to-target distance as seen in the thermogram inserted in Fig. 9. The graph in this figure shows the temperature profiles for the $0.2 \mathrm{ml}$ defect (the one having the lesser defect contrast) and a sound area close to it. As can be observed, heat was applied at three different cycles, following each time a spiral pattern to heat the area. At every cycle, the specimen was able to absorb more energy with respect to the water present inside the honeycomb core, increasing defect contrast in this way. Representative thermograms are also shown for each cycle, which lasted around 2 minutes each (100 to 130 s). As seen, this time was enough to create enough thermal contrast between defect and sound areas and to go back to equilibrium at the end of each cycle.

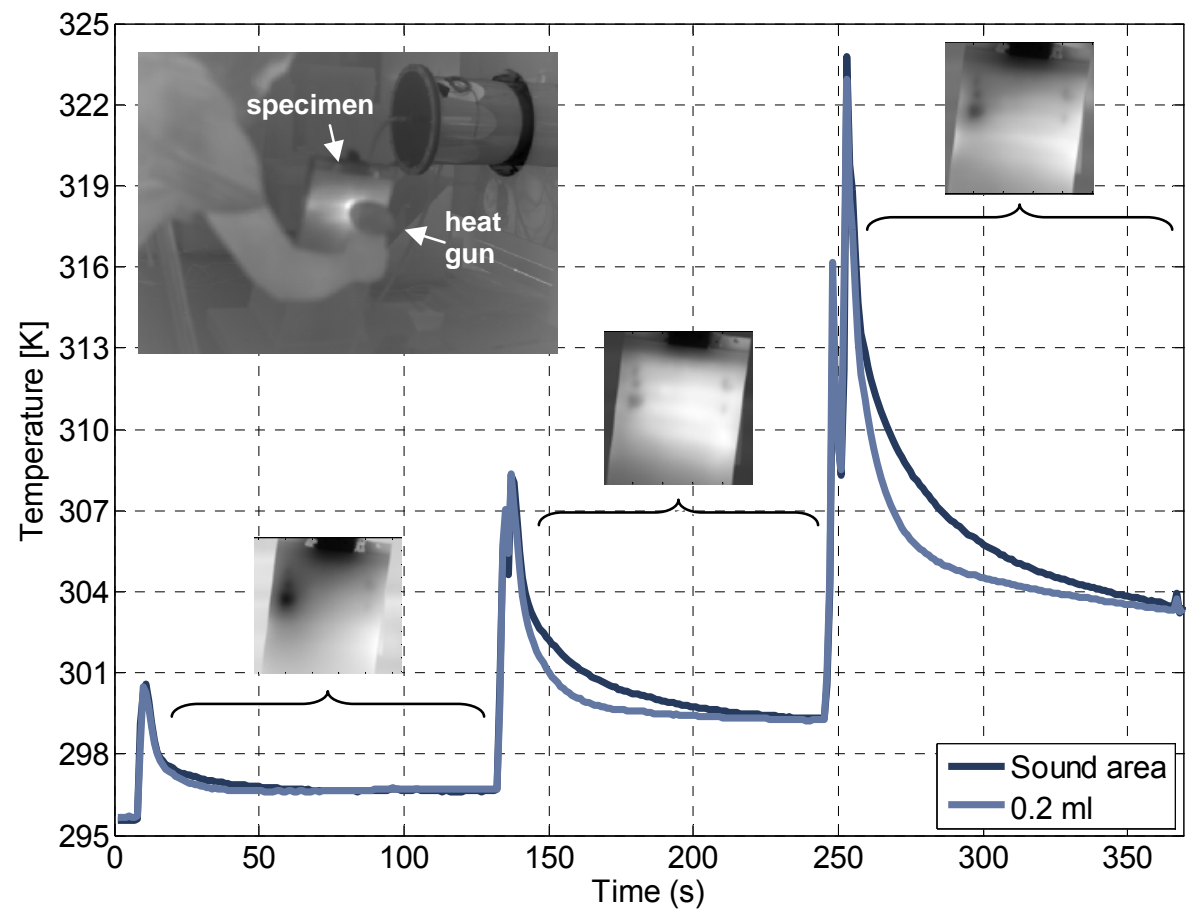

Fig. 9. Temperature profiles for the $0.2 \mathrm{ml}$ defect and the sound area after active manual heating using a heat gun, three heating cycles are shown (data from high definition camera).

Raw thermograms corresponding to the HD camera and the microbolometer are presented in Fig. 10a and d (first column), respectively. Defect contrast is greater for the HD camera, as expected but still it lacks of defect contrast. Signal processing was applied to raw data obtained with both cameras giving the results presented in the second and third columns of Fig. 10. As seen, it was possible to retrieve all for defects with both cameras after processing. The three defective areas at the left of the sample are perfectly seen in Fig. 10b, which was processed by differential absolute contrast (DAC) [17], and some light indications are observed in the microbolometer PCT result of Fig. 10e. Some indications of the carbon fibers (at $45^{\circ}$ ) can be seenFig. 10b. These features are observed with even better definition in the PCT result of Fig. 10c, showing that active stimulation allow to retrieve this information during this kind of inspection (manual inspection using a heat gun). Traces of non-uniform heating can also be devised inFig. 10b and c. The last image in Fig. 10f show a processed result from the microbolometer data. All defects are detected with more or less defect contrast.

These results show that it is possible to retrieve the presence of as little as $0.2 \mathrm{ml}$ of water more than 32 minutes after landing by actively inspecting the sample from a distance of $1.8 \mathrm{~m}$. Advanced signal processing techniques such as 
PCT, PPT and DAC is required in order to increase defect contrast. This is particularly important when using a microbolometer, which present a much lesser defect contrast.

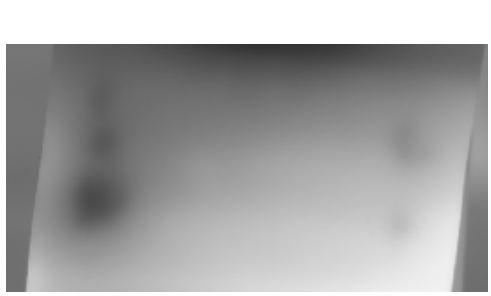

(a)

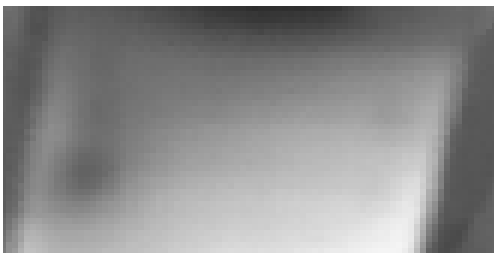

(d)

\section{HD camera results}

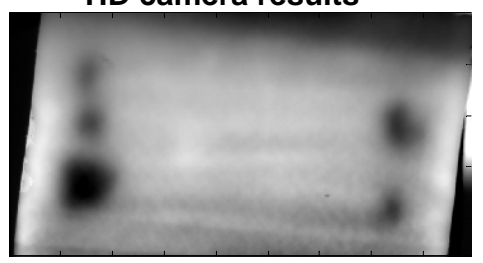

(b)

Microbolometer results

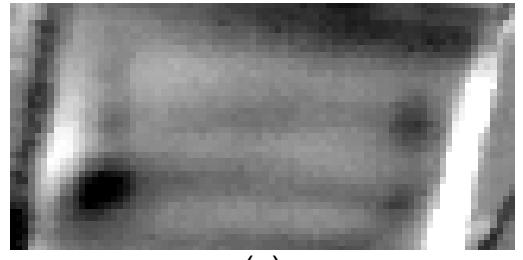

(e)

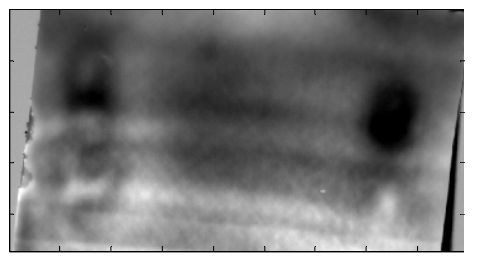

(c)

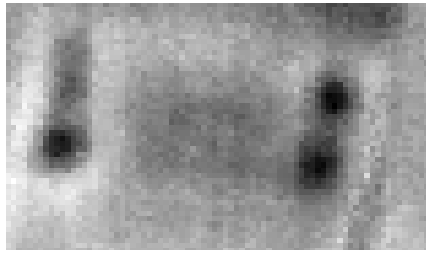

(f)

Fig. 10. Raw thermograms and data processing results active thermography using a heat gun with $1.8 \mathrm{~m}$ camera-to-target distance (second heating cycle in all cases unless indicated otherwise). Top row (high definition camera): (a) raw thermogram at $t=20 \mathrm{~s}$; (b) differential absolute contrast at $t=11 \mathrm{~s}$; (b) principal component thermography (EOF5, $3^{\text {rd }}$ heating cycle). Bottom row (microbolometer): principal component thermography $\left(2^{\text {nd }}\right.$ heating cycle) (c) EOF4; and (d) EOF6.

\subsubsection{Camera to target distance of $18 \mathrm{~m}$}

Active inspection was also undertaken to determine if is possible to improve defect detection at this distance. An infrared lamp was employed to heat up the surface, manually, as seen in Fig. 11a.

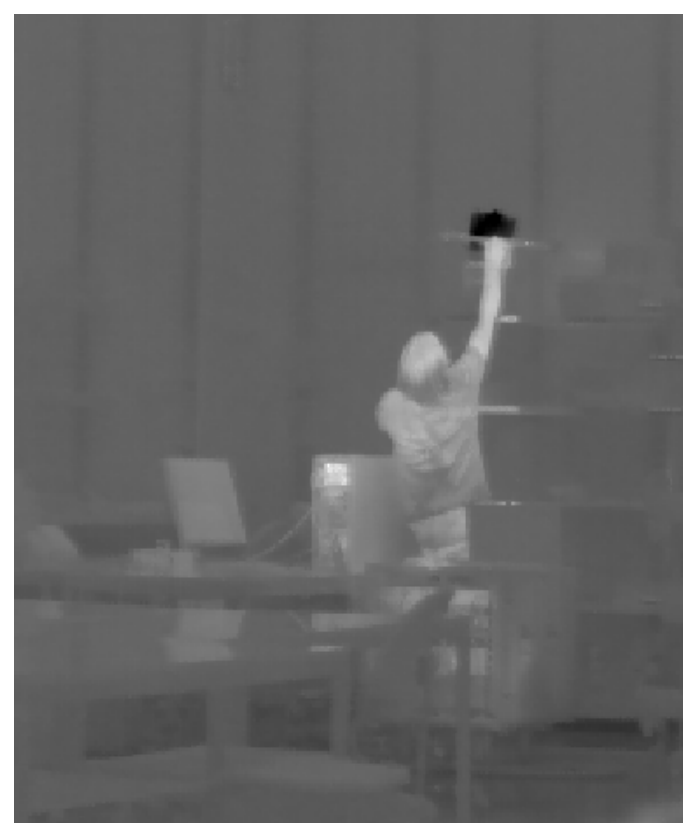

(a)

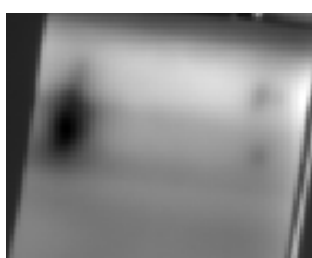

(b)

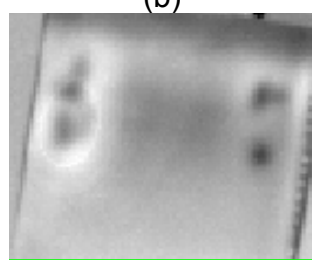

(c)

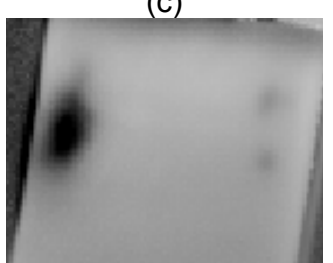

(d)

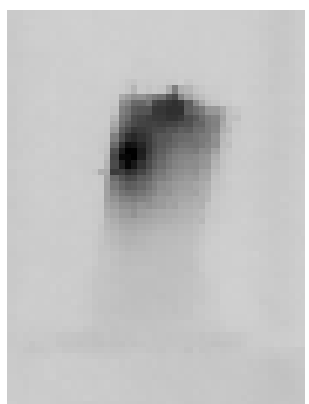

(e)

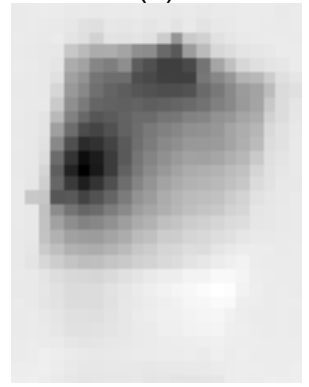

(f)

Fig. 11. Data processing results by manual active thermography using an infrared lamp (target at $18 \mathrm{~m}$ ) for high definition camera: (a) raw thermogram cropped to illustrate the manual heating procedure using an IR lamp; (b) and (c) principal component thermography: EOF2 and EOF6, respectively; and (d) pulsed phase thermography at $f=0.03 \mathrm{~Hz}$. 
Even though, with the exception of Defect $\mathrm{E}$ (with $9 \mathrm{ml}$ of water), no other defect was visible in passive mode at the end of the first experiment, active stimulation with advanced signal processing allowed to detect all four defects using the HD camera. Some traces of non-uniform heating, due to manual manipulation of the heating source, can be slightly seen in Fig. $11 \mathrm{~b}$, obtained by PCT. Defects are better resolved in Fig. 11c (also obtained by PCT), in which it is also possible to observe that there are actually three defect regions on the left side (where Defects $A$ and $E$ are located), giving more weight to the hypothesis of water migration in this area. Pulsed phase thermography (PPT) [18] was also applied to this thermographic sequence. The result (Fig. 11d) is quite similar to PCT's EOF2 of Fig. 11b, but without the traces from the heating source. This is an interesting well-known characteristic of phase results from PPT [19]. In the case of microbolometer results, it was not possible to clearly detect any defect. A gros indication of defect $E$ (the one containing the largest amount of water) can be seen in the raw thermogram of Fig. 11e, but defect contrast is not improved after processing as shown in Fig. 11f.

Hence, it is clear that spatial resolution plays a crucial role on defect detection of small areas containing low quantities of water. The use of active stimulation and advanced processing do not seems to improve detectability in these cases.

\section{Conclusions}

Passive thermography constitutes and NDT technique worthy of consideration for inspecting aircraft composed of honeycomb sandwich structures susceptible to water detection. It is concluded from this research work that the best moment to detect and quantify defects is within a 30 minutes time window after landing, in order to maximize thermal contrast within defective areas (with frozen water) and non-defective area. In such a case, it is possible to detect water quantities as low as $0.2 \mathrm{ml}$ using either a HD camera or a microbolometer. While defect detection is possible from a distance of 18 from the target and without processing using a HD camera, defect detection under these conditions is only possible for water quantities of, roughly, $1 \mathrm{ml}$ of more using a microbolometer and advance processing techniques.

It is worth noting as well that, in order to apply advanced signal processing it is necessary to acquire thermogram sequences in static configuration, which means that the aircraft needs to be on a halt. This has a direct implication on the time window for defect detection by passive thermography, which is already limited to approximately 30 minutes for small defects. For times greater than this threshold, an interesting possibility is to use active thermography techniques.

A relatively easy to deploy active inspection procedure can be sought in order to increase defect detectability for times above 30 minutes after landing. In almost all cases (HD camera and microbolometer from 1.8 and $18 \mathrm{~m}$ distances) defect detection was improved, with the exception of the microbolometer at $18 \mathrm{~m}$ from the sample for which spatial resolution was simply not enough to retrieve thermal signatures for small defects (less than $1 \mathrm{ml}$ of water).

\section{REFERENCES}

[1] Li C., Ueno R. and Lefebvre V., "Investigation of an accelerated moisture removal approach of a composite aircraft control surface," Proc. 38th SAMPE Fall Technical Conference: Global Advances in Materials and Process Engineering, November 6-9 (2006).

[2] Campbell F. C., "The case against honeycomb core," Proc. International SAMPE Symposium and Exhibition, 49, 3680-3688 (2004).

[3] Shafizadeh J. E, Seferis J. C., Chesmar E. F., and Geyer R., "Evaluation of the in-service performance Behaviour honeycomb composite sandwich structures," Journal of Materials Engineering and Performance, 8(6), 661-668 (1999).

[4] Radtke T. C., Charon A. and Vodicka R., "Hot/wet environmental degradation of honeycomb sandwich structure representative of F/A- 18: Flatwise Tension Strength," DSTO Aeronautical and Maritime Research Laboratory, (1999).

[5] Giguère J. S. R., "Damage mechanisms and non-destructive testing in the case of water ingress in CF-18 flight control surfaces," Technical Memorandum, DCIEMTM 2000-098, August (2000).

[6] Davis M. J., Chester R. J., Perl D. R., Pomerleau E. and Vallerand M., "Honeycomb bond and core durability issues; experiences within CREDP nations," Aging Aircraft Conference, Williamsberg, VA, August 31st-September 2nd (1998).

[7] Rahman A., "Effect of nodebond failures on structural integrity of F/A 18 trailing edge flaps," 9th Joint FAA/DoD/NASA Aging Aircraft Conference, Kansas City, MO, May 4-7 (2009).

[8] Balaskó M. Sváb E., Molnár Gy., and Veres I., "Classification of defects in honeycomb composite structure of helicopter rotor blades," Nuclear Instruments and Methods in Physics Research A, 542, 45-51(2005).

[9] Shafizadeh J. E., Seferis J. C., Chesmar E. F. and Geyer R., "Evaluation of the In-Service Performance Behavior of Honeycomb Composite Sandwich Structures," Journal of Materials Engineering and Performance, 8(6), 661-668 (1999). 
[10] Helium leak detection on CF-18 Rudder, Bombardier Aerospace Defence Services, Contract No. SES DI 2401,24 February (2000).

[11] Edwards A. K., Savage S., Hungler P. L. and Krause T. W. "Examination of F/A-18 honeycomb composite rudders for disbond due to water using through-transmission ultrasonics," Ultragarsas (Ultrasound), 66(2), 36-44 (2011).

[12] Swiderski W., "Nondestructive Testing of Honeycomb Type Composites by an Infrared Thermography Method," Proc. IV Conferencia Panamericana de END, Buenos Aires, Octubre (2007).

[13] Vavilov V., Kilimov A., Nesteruk D. and Shiryaev V., "Detecting water in aviation honeycomb structures by using transient IR thermographic NDT," Proc. ThermoSense XXV, 5073, 345-355 (2003).

[14] Vavilov V. and Nesteruk D., "Evaluating water content in aviation panels by transient IR thermography," Proc. ThermoSense XXVII, 5782, 411-417 (2005).

[15] FLIR EMEA, "Using a thermal imaging camera to perform water ingress inspections," 14 September 2011, $<$ http://www.youtube.com/watch?v=ZEXVaoxbp2U> (22 March 2012).

[16] Rajic N., "Principal component thermography for flaw contrast enhancement and flaw depth characterisation in composite structures," Compos. Struct., 58:521-528, 2002.

[17] Pilla M., Klein M., Maldague X. and Salerno A., "New Absolute Contrast for Pulsed Thermography," QIRT 2002, D. Balageas, G. Busse, G. Carlomagno eds., Proc. of QIRT pp. 53-58, 2002.

[18] Maldague X. P. and Marinetti S. "Pulse Phase Infrared Thermography," J. Appl. Phys., 79(5):2694-2698, 1996.

[19] Ibarra-Castanedo C. and Maldague X. "Pulsed Phase Thermography Reviewed," QIRT J., 1(1):47-70, 2004. 